\title{
The Loroxanthin Cycle: A New Type of Xanthophyll Cycle in Green Algae (Chlorophyta)
}

\author{
Tomas E. van den Berg ${ }^{\dagger}$ and Roberta Croce* \\ Department of Physics and Astronomy, Faculty of Science, Vrije Universiteit Amsterdam, Amsterdam, Netherlands
}

OPEN ACCESS

Edited by:

Thomas Roach,

University of Innsbruck, Austria

Reviewed by:

Renata Szymańska,

AGH University of Science and

Technology, Poland

Laurie Leonelli,

University of Illinois at Urbana-

Champaign, United States

*Correspondence:

Roberta Croce

r.croce@vu.n

${ }^{\dagger}$ Present address:

Tomas E. van den Berg,

Integrated Devices and Systems

Group, University of Twente,

Enschede, Netherlands

Specialty section:

This article was submitted to

Plant Physiology,

a section of the journal

Frontiers in Plant Science

Received: 18 October 2021

Accepted: 18 January 2022

Published: 17 February 2022

Citation:

van den Berg TE and Croce R (2022)

The Loroxanthin Cycle: A New Type

of Xanthophyll Cycle in Green Algae

(Chlorophyta).

Front. Plant Sci. 13:797294.

doi: 10.3389/fpls.2022.797294
Xanthophyll cycles $(X C)$ have proven to be major contributors to photoacclimation for many organisms. This work describes a light-driven $\mathrm{XC}$ operating in the chlorophyte Chlamydomonas reinhardtii and involving the xanthophylls Lutein (L) and Loroxanthin (Lo). Pigments were quantified during a switch from high to low light (LL) and at different time points from cells grown in Day/Night cycle. Trimeric LHCll was purified from cells acclimated to high or $L L$ and their pigment content and spectroscopic properties were characterized. The $L O /(L+L O)$ ratio in the cells varies by a factor of 10 between cells grown in low or high light $(\mathrm{HL})$ leading to a change in the $\mathrm{Lo} /(\mathrm{L}+\mathrm{LO})$ ratio in trimeric $\mathrm{LHCll}$ from .5 in low light to .07 in HL. Trimeric LhcbMs binding Loroxanthin have $5 \pm 1 \%$ higher excitation energy (EE) transfer (EET) from carotenoid to Chlorophyll as well as higher thermo- and photostability than trimeric LhcbMs that only bind Lutein. The Loroxanthin cycle operates on long time scales (hours to days) and likely evolved as a shade adaptation. It has many similarities with the Lutein-epoxide - Lutein cycle (LLx) of plants.

Keywords: xanthophyll cycle, loroxanthin cycle, NPQ, Lutein, Chlamydomonas reinhardtii, LHC, photoacclimation

\section{INTRODUCTION}

Green algae are found worldwide in a large variety of habitats: from the dessert crust (Perera et al., 2018) to the pole-ice (Kirst and Wiencke, 1995). Their ability to grow under different conditions follows from the millions of years of evolution after their ancestor encapsulated a cyanobacterium. Their evolutionary success did not rely on the photosynthetic electron transfer chain that has remained largely unchanged but instead depended on the versatile acclimation machinery (Ballottari et al., 2012; Leliaert et al., 2012; de Vries and Archibald, 2018).

In all organisms performing oxygenic photosynthesis, light-harvesting and trapping of excitation energy (EE) occur in photosystems (PS) I and II. The absorption cross-section of the PS core complexes is extended by an outer antenna, which in plants and green algae is composed of members of the light-harvesting multigenic family (Croce and van Amerongen, 2020; Pan et al., 2020). LHCII is the main antenna complex in plants and green algae, it can be associated with both photosystems and is mainly present in trimeric form. In the Chlorophyte Chlamydomonas reinhardtii, LHCII is 
composed of nine LHCBM proteins (Minagawa and Takahashi, 2004; Natali and Croce, 2015). In addition to LHCII, C. reinhardtii contains PSI-specific antennae, called Lhca (Mozzo et al., 2010) and the monomeric antennae CP26 and CP29, mainly associated with PSII (Elrad et al., 2002). Four carotenoid binding sites are present in LHCII, that are highly conserved in plants and green algae (Figure 1). The N1 site is highly specific for Neoxanthin in most complexes. The L1 site is usually occupied by Lutein (L) and the L2 site also preferentially binds L (Natali and Croce, 2015; Pan et al., 2020). Studies on the LHCII of plant showed that the xanthophylls in all three bindings sites are involved in light-harvesting (Croce et al., 2000), while only L1 and L2 are responsible for Chlorophyll (Chl) triplet quenching (Mozzo et al., 2008). The fourth carotenoid binding site, V1, is located at the periphery of the complex and is occupied either by Violaxanthin or Lutein. Experiments on plants have shown that in this pocket, the carotenoid is only weakly bound to the complex, not involved in light-harvesting and is easily lost during the isolation of the complex from the membrane (Caffarri et al., 2001).

A large variety of carotenoids is present in plants and green algae (Takaichi, 2011; Jeffrey et al., 2011) acting as light sensors, shading pigments, antioxidants, membrane stabilizers, lightharvesting pigments, and excitation-energy quenchers (Stange, 2016). A selection of carotenoids active in light-harvesting and photoprotection is associated with photosynthetic proteins. $\beta$-carotene (BC) is mainly bound to the PS I and II core, while xanthophylls are bound to the light-harvesting complexes of plants and green algae (Caffarri et al., 2014).

The light-harvesting antennae are involved in multiple lightacclimation processes. These are vital for plants and green algae because they allow the fine-tuning of photosynthesis,

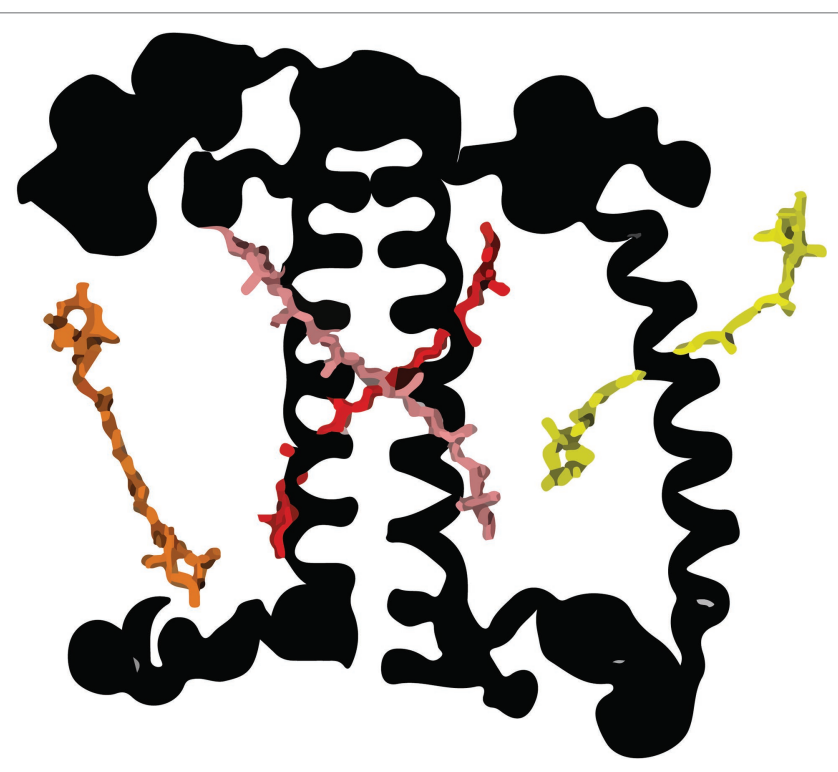

FIGURE 1 | The carotenoid binding sites of LHCll monomer (from Shen et al., 2019). Neoxanthin in the N1 site (yellow), Lutein (L) in the L1 (red) and L2 (pink) sites, and Violaxanthin in the V1 site (orange) were modeled according to the structure of plant LHCII (Liu et al., 2004). For clarity, the chlorophylls (Chl) are omitted. preventing photodamage or photo-starvation. The acclimation processes occur on different time-scales as responses to changes in light quality and quantity (Wobbe et al., 2016): from shortterm processes that act on seconds to minutes to long-term processes lasting for hours to days. Long-term processes are connected to regulated changes in gene expression that affect the capacity of photosynthetic electron transport, respiration, and light-harvesting (Falkowski and Chen, 2003), $\mathrm{CO}_{2}$ concentrating mechanisms and $\mathrm{CO}_{2}$ fixation (Giordano et al., 2005), as well as the capacity for photoprotection and shading (Wobbe et al., 2016). Short-term processes include non-photochemical quenching (NPQ), a process that reduces the excited-state lifetime of the chlorophylls, thus preventing Chl triplet formation that can lead to the production of damaging reactive oxygen species (ROS; Bassi and Dall'Osto, 2021). NPQ is activated by the acidification of the lumen. In C. reinhardtii, several proteins are involved in the fast phase of NPQ called qE (s to min): LHCSR1 and LHCSR3 (Peers et al., 2009; Dinc et al., 2016) and PsbS (Correa-Galvis et al., 2016; Tibiletti et al., 2016). In addition, some algae contain the Xanthophyll cycles (XC), which have multiple roles in short and long-term photoprotection (García-Plazaola et al., 2007).

Thus far, six such cycles are known (García-Plazaola et al., 2007). The most extensively studied are the Violaxanthin Antheraxanthin - Zeaxanthin cycle (VAZ) present in plants and green algae, the Diadinoxanthin - Diatoxanthin cycle (Ddx) present in diatoms and the Lutein-epoxide - Lutein cycle (LLx) present in some plants. Out of the other three cycles two are truncated versions of the VAZ cycle, namely the Violaxanthin - Antheraxanthin cycle (VA; Goss et al., 1998; Stamenković et al., 2014) and the Antheraxanthin - Zeaxanthin (AZ) cycle (Rmiki et al., 1996). The final one is the Lutein - Siphonaxanthin (LS) cycle (Raniello et al., 2006; for more details see GarcíaPlazaola et al., 2007). Most cycles consist of the de-epoxidation of xanthophylls in (light) stress and their epoxidation in the absence of stress [low light (LL) or darkness; Goss and Latowski, 2020; Fernández-Marín et al., 2021]. The epoxy- and epoxyfree xanthophylls have different properties that favor lightharvesting or photoprotection (Havaux, 1998). The VAZ cycle and the Ddx cycle are well-known to contribute to NPQ (Goss and Jakob, 2010). A role in NPQ has also been suggested for the LLx cycle (Esteban et al., 2010; Matsubara et al., 2011; Leonelli et al., 2017). The activation of the xanthophyll-dependent NPQ requires several minutes and it is thus slower than the LHCSR/PSBS dependent $\mathrm{qE}$, but still very fast compared to other acclimation processes (Stamenković et al., 2014; Quaas et al., 2015; Christa et al., 2017). In addition to their role in $\mathrm{NPQ}$, the XCs protect from photoinhibition in high light (HL) by enhancing the antioxidant activity in the membrane (Havaux and Niyogi, 1999; Havaux et al., 2007; Johnson et al., 2007; Lepetit et al., 2010) and the membrane stability (Havaux, 1998; Gruszecki and Strzałka, 2005; Bojko et al., 2019). Upon a switch to LL the XCs provide advantages in light-harvesting by decreasing the energy losses through NPQ (VAZ; Kromdijk et al., 2016) and increasing the carotenoid to Chl excitation energy transfer (EET; LLx; Matsubara et al., 2007; Leonelli et al., 2017). All XCs are active upon a change of light intensity 
but the turnover kinetics can vary from minutes (VAZ, Ddx) to days (LLx) depending on the type of cycle (García-Plazaola et al., 2007; Goss and Jakob, 2010), the plant species (LLx; García-Plazaola et al., 2007), temperature (VAZ; Reinhold et al., 2008) and the changes in light intensity (VAZ; Kress and Jahns, 2017). Additionally, the XC pool size can be highly variable, ranging from a minor fraction to the dominating xanthophylls (García-Plazaola et al., 2007; Stamenković et al., 2014) and not all xanthophylls in the pool may be available to the cycle (Snyder et al., 2005). Lastly, XCs can influence the composition of the free pool of xanthophylls in the membrane, the xanthophylls bound to the light-harvesting antenna or both, depending on the cycle, the species and the duration of the stress condition (Snyder et al., 2005; Matsubara et al., 2007; Reinhold et al., 2008; Xu et al., 2015).

The unicellular green alga C. reinhardtii became a model green alga because it grows quickly in the lab and is easy to cross (Sasso et al., 2018). Its PS and their antenna (Mozzo et al., 2010; Natali and Croce, 2015; Shen et al., 2019; Suga et al., 2019; Huang et al., 2021) and photoacclimation behavior (Bonente et al., 2012; Allorent et al., 2013; Nawrocki et al., 2016, 2020; Polukhina et al., 2016) are well characterized. Interestingly, although the VAZ XC was observed in this alga, this cycle is not involved in NPQ (Bonente et al., 2011; Quaas et al., 2015).

One of the xanthophylls of $C$. reinhardtii is Loroxanthin (Lo), which is formed by the hydroxylation of the methyl group at $\mathrm{C} 9$ of the polyene of Lutein by an unknown enzyme (Grossman et al., 2004). It is widespread among Chlorophyte, Euglenophyte, and Chlorarachniophyte (Takaichi, 2011) and it binds to the light-harvesting complexes (Bishop, 1996; Mozzo et al., 2010; Natali and Croce, 2015; van den Berg et al., 2018). Loroxanthin is associated with the PSI and PSII supercomplexes of low light-grown C. reinhardtii (Pineau et al., 2001; Drop et al., 2011, 2014). Furthermore, Loroxanthin abundance and its ratio with Lutein vary in different light intensities in $C$. reinhardtii and other Chlorophyte (Bishop et al., 1989; Garrido et al., 2009; Bonente et al., 2012; van den Berg et al., 2019). We hypothesize that the differences in cellular Lutein and Loroxanthin content observed in low and HL grown cultures are due to a light-driven XC (Figure 2A, spectra in Figure 2B) that affects the Xanthophyll composition of the light-harvesting complexes and thereby their properties. In this work, we tested this hypothesis using a combination of biochemical and spectroscopic analyses at the protein and cellular levels.

\section{MATERIALS AND METHODS}

\section{Growing Conditions}

Chlamydomonas reinhardtii CC-124 was grown photoautotrophically in high salt medium (HSM; Sueoka, 1960) in LL $\left(15 \mu \mathrm{mol} \mathrm{m}^{-2} \mathrm{~s}^{-1}\right.$ white fluorescent bulbs) or $\mathrm{HL}(500 \mu \mathrm{mol}$ $\mathrm{m}^{-2} \mathrm{~s}^{-1}$ white fluorescent bulbs) shaking at $150 \mathrm{rpm}$ at room temperature (RT) or it was grown in a DN cycle [18D:6 N,

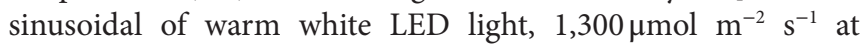
midday (9h)] in a bubble column photobioreactor (PSI, Czech Republic) at $25^{\circ} \mathrm{C}$. Biological replicates consist of independent
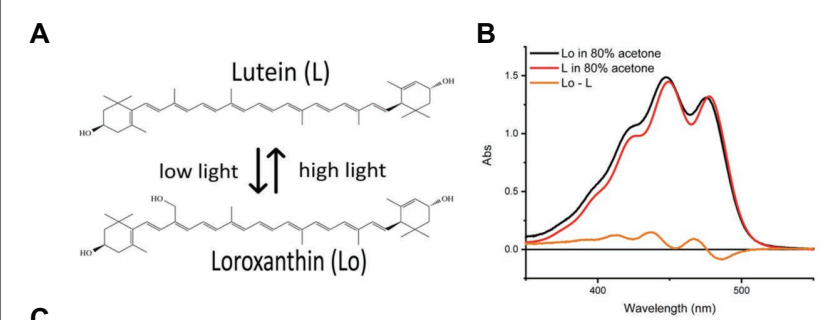

C

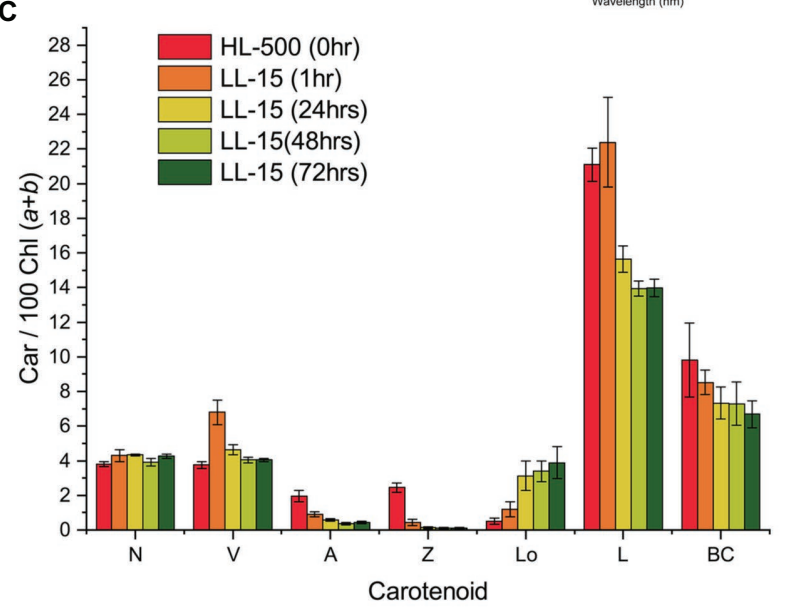

D
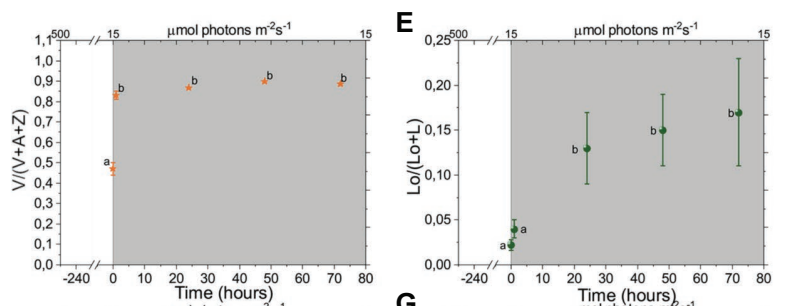

$\mathbf{F}$
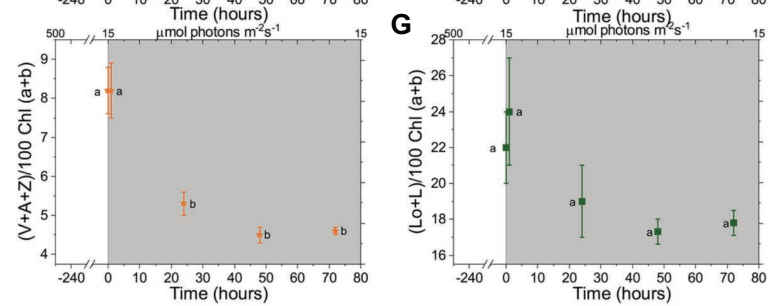

FIGURE 2 | Spectra of Loroxanthin (LO) and Lutein and the changes in their content at different light intensities. (A) Chemical structures of $L$ and $L o$ and an illustration of their relationship at different light intensities. (B) Absorption spectra of Loroxanthin and Lutein in $80 \%$ acetone. The difference spectrum is also shown. (C) Carotenoid composition [relative to $100 \mathrm{Chl}(a+b)]$. The number in the legend indicates the light intensity in $\mu \mathrm{mol}$ photons $\mathrm{m}^{-2} \mathrm{~s}^{-1}$.

(D) Violaxanthin - Antheraxanthin - Zeaxanthin cycle (VAZ) epoxidation state, (E) LLo hydroxylation state and VAZ (F) as well as LLo (G) pool size [relative to $100 \mathrm{Chl}(a+b)]$ of Chlamydomonas reinhardtii cells at different time points $(0,1,24,48$, and $72 h)$ following the shift of the culture from continuous $H L$ to continuous LL (D-G, gray area). Averages represent two technical replicates per five biological replicates per timepoint. Error bars represent the SE. Letters $(a, b)$ indicate significant differences between groups (ANOVA). N, Neoxanthin; V, Violaxanthin; A, Antheraxanthin; Z, Zeaxanthin; Lo, Loroxanthin; L, Lutein; and BC, $\beta$-carotene.

cultures that were acclimated for at least 10 generations at each culturing condition ( $\mathrm{D} / \mathrm{N}$ or prior to transfer to a different light intensity) with culture dilution every $2-3$ days, maintaining the cells in the exponential growth phase. 


\section{Purification of Antenna Complexes}

Thylakoids were prepared as previously described (Chua and Bennoun, 1975) with the addition of $1 \mathrm{mM}$ benzamidine and $\varepsilon$-aminocaproic acid to the buffer (Tricine $\mathrm{NaOH}$ pH 7.8 instead of Hepes $\mathrm{pH}$ 7.5). In short, the thylakoid membranes were purified using a discontinuous sucrose gradient $(100,000 \times g$, $1 \mathrm{~h}, 4^{\circ} \mathrm{C}$ in a SW41 swinging bucket rotor). Thylakoids were pelleted, unstacked with $5 \mathrm{mM}$ EDTA, washed with $10 \mathrm{mM}$ tricine- $\mathrm{NaOH}$ ( $\mathrm{pH}$ 7.8), and homogenized in the solubilization buffer (10 mM Tricine-NaOH, $150 \mathrm{mM} \mathrm{NaCl}, \mathrm{pH} 7.8 \mathrm{pH})$. Subsequently, thylakoids (kept in the dark on ice) diluted at a Chl concentration of $1.0 \mathrm{mg} \mathrm{ml}^{-1}$ were mixed with an equal volume of solubilization buffer containing freshly prepared detergent [0.6\% Dodecyl- $\alpha$-d-maltoside ( $\alpha$-DM; Anatrace)]. The thylakoids were solubilized for $20 \mathrm{~min}$ at $4^{\circ} \mathrm{C}$ in the dark with end-over-end shaking. Isolation of complexes was performed by sucrose density gradient made by freezing and thawing $.5 \mathrm{M}$ sucrose, $10 \mathrm{mM}$ Tricine- $\mathrm{NaOH}, 0.05 \% \quad \alpha-\mathrm{DM}(\mathrm{pH} 7.8)$ centrifuged at $240,000 \times g$ for $17 \mathrm{~h}$ at $4^{\circ} \mathrm{C}$ in a SW41 rotor. Green bands were collected with a syringe, flash-frozen in liquid nitrogen and stored at $-80^{\circ} \mathrm{C}$ until use.

\section{Steady-State Absorption, Fluorescence, Circular Dichroism, and Thermostability}

The sample buffer used for all RT experiments was .5 M sucrose, $20 \mathrm{mM}$ Tricine ( $\mathrm{pH} 7.8$ ), and .05\% alpha-DM. About $66 \%$ $(\mathrm{w} / \mathrm{w})$ glycerol was added to the buffer for $77 \mathrm{~K}$ experiments. Sample optical density (OD) at the maximum in the Qy region was .8-1 for absorption and circular dichroism (CD) and below .05 for fluorescence measurements. RT and $77 \mathrm{~K}$ absorption spectra were recorded with a Cary 4000 spectrophotometer (Varian) with a spectral bandwidth of $2 \mathrm{~nm}$. For $77 \mathrm{~K}$ measurements samples were cooled in a cryostat (Oxford Instruments). About $77 \mathrm{~K}$ absorption spectra were measured with a UV-2600 spectrophotometer (Shimadzu) with a spectral bandwidth of $2 \mathrm{~nm}$. Fluorescence emission spectra at RT and $77 \mathrm{~K}$ were recorded on a Fluorlog 3.22 spectrofluorimeter (Jobin-Yvon spex). For fluorescence emission spectra, the spectral bandwidths were $3 \mathrm{~nm}$ for excitation (440, 475, and $500 \mathrm{~nm}$ ), and $1 \mathrm{~nm}$ for emission. Excitation spectra were recorded at $735 \mathrm{~nm}$ emission with the spectral bandwidths $1 \mathrm{~nm}$ for excitation, and $3 \mathrm{~nm}$ for emission. Around $735 \mathrm{~nm}$ was chosen in order to record the excitation spectra up to $700 \mathrm{~nm}$. Excitation spectra at 680 and $735 \mathrm{~nm}$ were identical. An optical filter was placed before the detector to block light $<600 \mathrm{~nm}$ for emission or $<700 \mathrm{~nm}$ for excitation spectra. CD spectra were recorded at $20^{\circ} \mathrm{C}$ with a Chirascan CD spectrophotometer (Applied Photophysics) equipped with a temperature control unit TC125 (Quantum Northwest). The spectral bandwidth was $1 \mathrm{~nm}$ and the sample volume $400 \mu$ l. The thermostability of the complexes was determined by the loss of the CD signal between 450 and $550 \mathrm{~nm}$ as a function of increasing temperature $\left(20-90^{\circ} \mathrm{C}\right.$, $2.5^{\circ} \mathrm{C}$ steps). Each heating step took $1 \mathrm{~min}$, followed by $1 \mathrm{~min}$ of equilibration before measuring the spectrum. Three biological replicates for all experiments, except $77 \mathrm{~K}$ absorption with two biological replicates.

\section{Pigment Analyses}

The pigment composition was determined by fitting the absorption spectrum of the $80 \%$ acetone extracts with the spectra of the individual pigments in the same solvent as earlier described (Croce et al., 2002) and by HPLC. In brief, pelleted cells were vortexed with $80 \%$ acetone, centrifuged, and if pellets were white supernatant was used for analyses. HPLC was performed on a System gold 126 equipped with a 168 UV-VIS detector (Beckman Coulter, United States) using a C18-Sphereclone column (Phenomenex 5U ODS1, 00G-4143E0, $4.6 \mathrm{~mm} \times 250 \mathrm{~mm}$ ). Loroxanthin, Neoxanthin, Violaxanthin, and Chl $b$ were separated according to an earlier described protocol (Pineau et al., 2001). Because with this method the separation between Chl $a$ and Lutein could not be achieved on our column, Violaxanthin, Antheraxanthin, Zeaxanthin, Lutein, $\mathrm{Chl} b, \mathrm{Chl} a$, and $\mathrm{BC}$ were additionally separated using another protocol (van den Berg et al., 2019). Pigment extracts from Arabidopsis LHCII were used as pigment calibration standard. Two technical replicates per five biological replicates per timepoint for the cultures transferred from high light to low light conditions. Two technical replicates per three biological replicates per timepoint for the DN cycle experiments. Two technical replicates per four biological replicates for the isolated LHCII samples.

\section{Time-Resolved Fluorescence}

Time-resolved fluorescence was measured at RT by a time-correlated single-photon counting (TCSPC) setup (FluoTime 200 fluorometer, PicoQuant). Samples were stirred with a magnetic bar in a $1 \mathrm{~cm}$ quartz cuvet. Excitation was performed with a laser diode at $438 \mathrm{~nm}$, with $5 \mathrm{MHz}$ repetition rate and $1 \mu \mathrm{W}$ power. Careful checks at higher and lower power confirmed the absence of non-linear processes (e.g., annihilation). Fluorescence was detected with 4 ps timesteps, at $680 \mathrm{~nm}(8 \mathrm{~nm}$ bandwidth), at an angle of $90^{\circ}$ with the excitation, through a polarizer set at the magic angle relative to the excitation polarization. The instrument response function (FWHM $88 \mathrm{ps}$ ) was determined using pinacyanol iodide in methanol (6ps lifetime; Van Oort et al., 2008). Data were accumulated until the number of counts in the peak channel was 20,000. Fluorescence decay curves were fitted with a multiexponential decay, with amplitudes and lifetimes convoluted with the IRF with the Fluofit software (Pico-Quant). Three components were necessary to get a good fit of the data as judged by $\chi^{2}$, the distribution of the residuals around 0 and the autocorrelation function of the residuals. Four biological replicates per sample.

\section{Carotenoid EET Efficiency}

Energy transfer efficiencies from Car-to-Chl a were estimated by fitting the fluorescence excitation spectra and the 1-Transmission (1-T) spectra with the spectra of the individual pigments and comparing the contribution of the same pigment to the two spectra. The 1-T and the excitation spectrum were normalized to the fitted quantity of $\mathrm{Chl} a$ (100\% efficiency of Chl $a->$ Chl $a$ EET). Deconvolution of spectra in the $400-520 \mathrm{~nm}$ wavelength range was performed as described in Croce et al. (2000). Three biological replicates per sample. 


\section{LHC Photostability}

The photostability of the complexes was determined by following the loss of absorption between 350 and $750 \mathrm{~nm}$ as a function of illumination time. The OD of the sample at $435 \mathrm{~nm}$ was .45 and the volume $500 \mu$ l. Samples were measured in a quartz cuvette with $1 \mathrm{~cm}$ path length. Samples were illuminated with white light from a halogen lamp $\left(4,500 \mu \mathrm{mol} \mathrm{m} \mathrm{m}^{-2} \mathrm{~s}^{-1}\right)$ equipped with an optical fiber arm through a $1 \mathrm{~cm}$ plastic cuvette filled with water and cooled by a fan to minimize heating by the light source during illumination. After each illumination time, the sample was mixed and an absorption spectrum was recorded. Two technical replicates per two biological replicates for each sample.

\section{Statistical Test}

Means were compared by paired, double-sided, students $t$-test, with the pairs representing the timepoints of individual biological replicates or in the case of LHCII, the individual preparations.

\section{RESULTS}

\section{Changes in Lutein and Loroxanthin Content Upon Transfer From High Light to Low Light}

To check the existence of a Lutein - Loroxanthin cycle in C. reinhardtii, we exposed the cells to changes in light intensity during growth and we measured the pigment content at different time points during light acclimation. The first set of experiments was performed on cells acclimated ( $>10$ days) to continuous HL $\left(500 \mu \mathrm{mol}\right.$ photons $\left.\mathrm{m}^{-2} \mathrm{~s}^{-1}\right)$ shifted to continuous LL $(15 \mu \mathrm{mol}$ photons $\mathrm{m}^{-2} \mathrm{~s}^{-1}$ ). In the second set of experiments, the cells were instead grown in a day/night cycle using (18:6h day/ night; $\mathrm{D} / \mathrm{N}$ ) a sinusoidal light regime (peak light intensity $1,300 \mu \mathrm{mol}$ photons $\mathrm{m}^{-2} \mathrm{~s}^{-1}$ ). The final sets of experiments, the purification and characterization of LHCII to asses the effects of the cycle on it, were performed on cells fully acclimated (>10 days) to LL or HL.

Pigment analysis (Figure 2C) showed that the $\mathrm{Lo} /(\mathrm{L}+\mathrm{Lo})$ ratio (Lutein hydroxylation state) increased eight times in the $72 \mathrm{~h}$ following the transfer from HL to LL (Figure 2E). In addition, the total $\mathrm{L}+\mathrm{Lo}$ pool size (normalized to the total $\mathrm{Chl}$ content, Figure 2G) decreased by $19 \%$ upon transfer to LL, but changes were not significant. The VAZ XC also showed changes during the experiment. The VAZ epoxidation state increased $\sim$ two times in the $72 \mathrm{~h}$ after the transfer from HL to LL (Figure 2D) and the total VAZ pool size was reduced by $44 \%$ (Figure 2F). The Chl concentration of the cultures transferred to LL increased 2.8 .5 times (Supplementary Figure S1). The $\mathrm{Chl} /$ Car ratio also increased from 2 to $2.6 \pm .2$ in $72 \mathrm{~h} \mathrm{LL}$, while the $\mathrm{Chl} a / b$ ratio was unaffected (Supplementary Figure S1).

\section{Lutein-Loroxanthin Changes During an 18:6 (D/N) Sinusoidal Light Regime}

To check if the changes in Lutein and Loroxanthin content also occurred in light conditions mimicking the natural environment, we monitored the levels of all xanthophylls during a simulated summer day $(18: 6 \mathrm{D} / \mathrm{N}$ cycle; Figure $3 \mathbf{A})$. The $\mathrm{Lo} /(\mathrm{L}+\mathrm{Lo})$ ratio varies throughout the day and roughly follows the inverse of the light intensity $\left(0-1,300 \mu \mathrm{mol} \mathrm{m} \mathrm{m}^{-2} \mathrm{~s}^{-1}\right.$; Figure $3 \mathrm{C})$. The $\mathrm{Lo} /(\mathrm{L}+\mathrm{Lo})$ ratio at any time during the $\mathrm{D} / \mathrm{N}$ cycle (Figure 3C) was more than $20 \%$ smaller than in the cultures shifted $(24 \mathrm{~h})$ from HL to LL (Figure 2E). The minimum of the $\mathrm{Lo} /(\mathrm{L}+\mathrm{Lo})$ ratio $(.02 \pm .01)$ was reached after 9-12 $\mathrm{h}$ of light and was delayed with respect to the de-epoxidation state of the VAZ cycle ( $\max 6-9 \mathrm{~h}$ ) (Figures 3B,C). Chl $a / b$ increased from $2.5 \pm .1$ to $2.8 \pm .1$ and $\mathrm{Chl} /$ Car decreased from $2.1 \pm .1$ to $1.9 \pm .1$ in the first $12 \mathrm{~h}$ of light and returned to the starting values at the end of the light period (Supplementary Figure S2).

In summary, the cellular content of Lutein and Loroxanthin is affected by changes in light intensity. The changes in Lo/ $(\mathrm{L}+\mathrm{Lo})$ ratio can be substantial when cells are adapted for days to different light conditions, but they are slow relative to the changes in the VAZ cycle.

\section{Lutein and Loroxanthin Content in Trimeric LHCII of High- or Low-Light Acclimated Chlamydomonas reinhardtii}

To investigate if the light-induced changes in Lutein and Loroxanthin content affect the pigment composition of LHCII, we purified trimeric LHCII from cultures that were photoacclimated to HL (LHCII-HL) or LL (LHCII-LL). The Lo/(L+Lo) ratio of the cells fully acclimated to low-light ( $>10$ days) was .49. Notice that this value is far higher than that observed upon $72 \mathrm{~h}$ of LL acclimation (Figure 2), indicating that that $72 \mathrm{~h}$ is not enough to reach a steady-state (Supplementary Table 2). The complexes were isolated by mildly solubilizing the thylakoid membranes and loading them on a sucrose density gradient. The pigment composition (Table 1) shows that while LHCII-LL binds a similar amount of Loroxanthin and Lutein (1.3 per monomer), LHCII-HL binds mainly Lutein (2.5 molecules per monomer) and contains only traces of Loroxanthin. The $86 \%$ decrease of Loroxanthin in LHCII-HL compared to LHCII-LL is compensated by an increase of Lutein. Other differences in pigment composition were a slightly higher $\mathrm{Chl}(a / b)$ ratio and a decrease of $\mathrm{V}$ in LHCII-HL compared to LHCII-LL.

\section{Spectral Characteristics of Trimeric LHCII Binding Lutein or Loroxanthin}

The absorption $(\mathrm{A}, \mathrm{B}), \mathrm{CD}(\mathrm{C})$, and fluorescence $(\mathrm{E}, \mathrm{F})$ spectra of LHCII-HL and LHCII-LL (Figure 4) display very similar characteristics. The small differences in absorption around $\sim 475$ and $\sim 650 \mathrm{~nm}$ for LHCII-LL compared with LHCII-HL reflect the difference in $\mathrm{Chl} a / b$ ratio. The lower absorption at $\sim 674 \mathrm{~nm}$ and higher at $\sim 665 \mathrm{~nm}$ and $\sim 680 \mathrm{~nm}$ at $77 \mathrm{~K}$ for LHCII-LL compared with LHCII-HL indicate small differences in the energy of some Chls a (Figures 4A,B). The higher absorption at $\sim 513 \mathrm{~nm}$ and lower at $\sim 497 \mathrm{~nm}$ for LHCII-LL compared with LHCII-HL, reflect changes in the carotenoid composition. This also explains the difference in the CD spectra in the $440-500 \mathrm{~nm}$ range (Figure 4C). The second derivative of the absorption spectra measured at $77 \mathrm{~K}$ (Figure 4D) demonstrates 
A

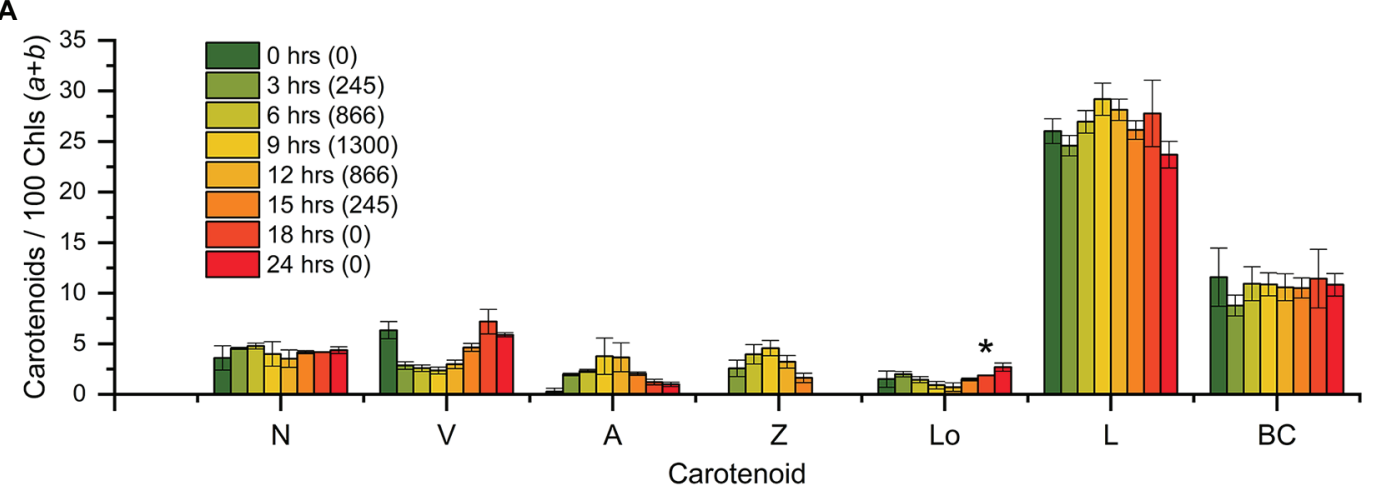

B

C
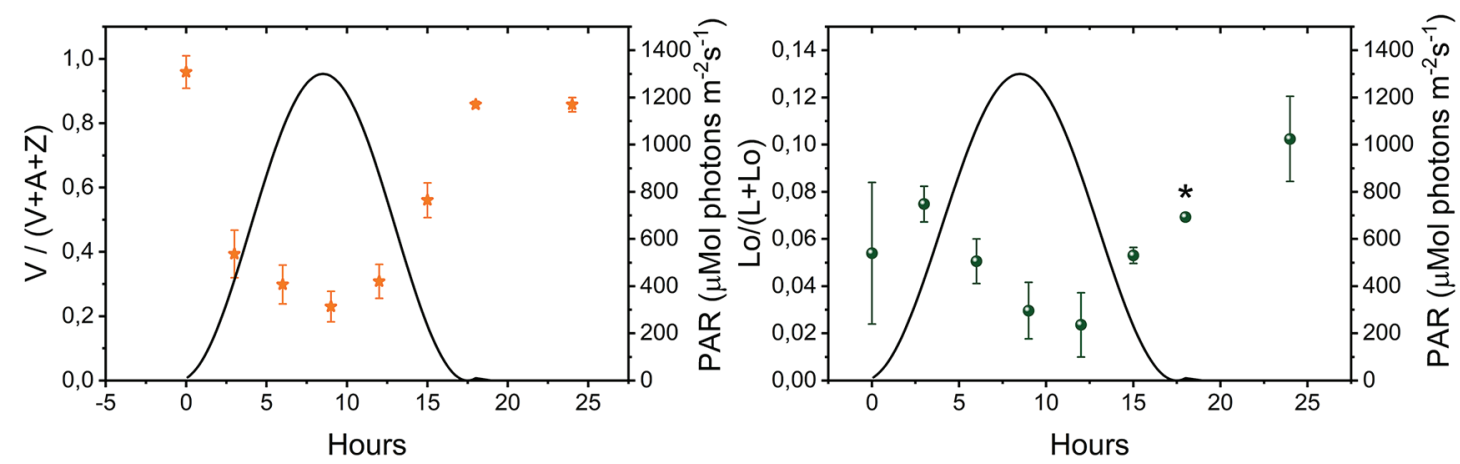

FIGURE 3 | Changes in the carotenoid composition of Chlamydomonas reinhardtii cells during a "simulated summer" day [18:6 (D:N) sinusoidal light regime]. (A) Carotenoid composition relative to $100 \mathrm{Chl}(a+b)$ molecules of cultures at different time points $(0,3,6,9,12,15,18$, and $24 \mathrm{~h}$, the number between brackets in the legend indicates the light intensity in $\mu \mathrm{mol}$ photons $\left.\mathrm{m}^{-2} \mathrm{~s}^{-1}\right)$. The sample at "O h" was taken right before dawn. The results are the mean of three biological replicates. The error bar represents the standard error of the mean (SE). (B) Epoxidation state of the VAZ pool. (C) Hydroxylation state of the L-Lo pool. N, Neoxanthin; V, Violaxanthin; A, Antheraxanthin; Z, Zeaxanthin; Lo, Loroxanthin; L, Lutein; and BC, $\beta$-carotene. Full statistics are shown in Supplementary Table 1. *indicate that data from one biological replicate for the protocol that separates neoxanthin and loroxanthin (see M\&M) is missing due to loss of sample ( $t=18)$, while replicates are available also for this time point for all other pigments and for the sum neoxanthin + loroxanthin.

TABLE 1 | Pigment composition of LHCIl-high light (HL) and LHCIl-low light (LL).

\begin{tabular}{|c|c|c|c|c|c|c|c|}
\hline LHCII & $C h l a / b$ & Chl/Car & $N$ & $\boldsymbol{V}$ & $A$ & Lo & $L$ \\
\hline LHCII-HL $(S E, n=4)$ & $1.29^{*}(0.05)$ & $3.78(0.13)$ & $0.6(0.1)$ & $0.25^{*}(0.03)$ & $0.08(0.13)$ & $0.18^{*}(0.06)$ & $2.5^{*}(0.1)$ \\
\hline
\end{tabular}

Xanthophyll content is normalized to $14 \mathrm{Chls}(a+b)$ molecules to indicate the xanthophylls per monomer in the trimeric complex. Chlorophylls (Chl) a/b and Ch//Car are expressed in moll mol. $n$ indicates the number of biological replicates. N, Neoxanthin; V, Violaxanthin; A, Antheraxanthin; Lo, Loroxanthin; and L, Lutein. "The means are significantly different $p<0.05$.

that both LHCII-LL and LHCII-HL have their lowest energy carotenoid transition around $\sim 510 \mathrm{~nm}$, similar to L2 in the trimeric LHCII of plants (Ruban et al., 2000).

\section{Lutein vs. Loroxanthin: Different Roles?}

Carotenoids in LHCs are important for the stability of the complexes (Paulsen et al., 1993) and are involved in light harvesting and photoprotection by quenching excited ${ }^{1} \mathrm{Chl}$ and ${ }^{3} \mathrm{Chl}$ as well as scavenging singlet oxygen (Frank et al., 2004). Time-resolved fluorescence measurements demonstrate that the different pigment composition of LHCII-HL and LHCII-LL does not affect their fluorescence lifetime (Table 2), indicating that the different content of Lutein and Loroxanthin does not influence the ${ }^{1} \mathrm{Chl}$ quenching, at least in vitro. Carotenoid to
Chl EET efficiency of LHCII-HL and LHCII-LL was tested by comparing the (1-T) spectrum with the fluorescence excitation spectrum. LHCII-LL has $5 \pm 1 \%$ higher Car- $>$ Chl EET efficiency than trimeric LHCII-HL (Figures 5A,B; Table 2). A higher Car- $>$ Chl EET efficiency (10\%) was also found for reconstituted LhcbM1 when compared with LhcbM1 reconstituted without Loroxanthin from an earlier work (Natali and Croce, 2015; Supplementary Figures S4, S6).

To investigate if the presence of Loroxanthin instead of Lutein influences the stability of LHCII, we followed the thermally-induced unfolding of LHCII-HL and -LL via CD measurements (Figure 5C). Additionally, we performed photostability experiments by following the bleaching kinetics of the LHCII absorption under high-light $(4,500 \mu \mathrm{mol}$ 


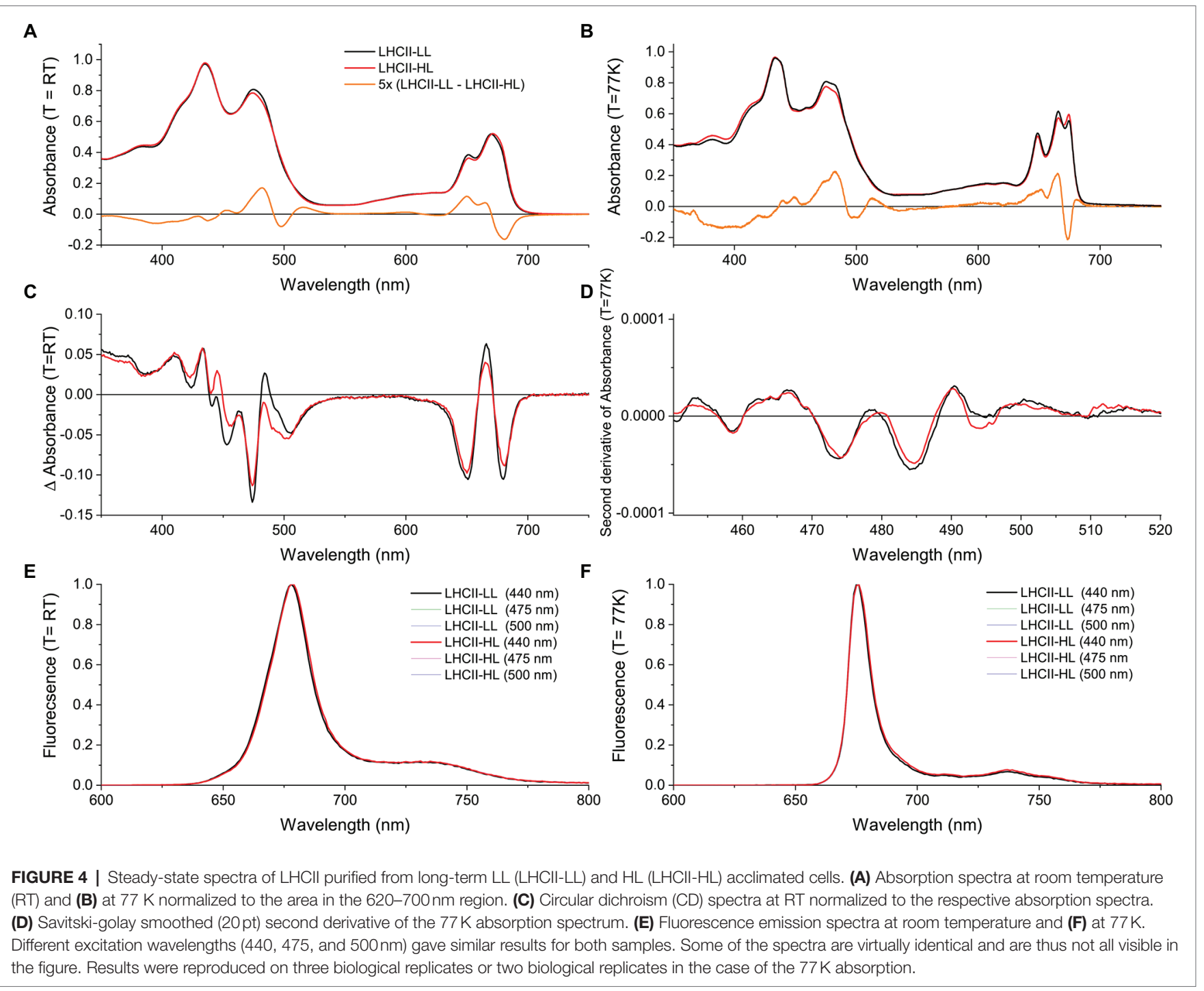

TABLE 2 | Properties of LHCII-HL and LHCII-LL.

\begin{tabular}{|c|c|c|c|c|}
\hline LHCII & $\begin{array}{l}\text { Average fluorescence } \\
\text { lifetime (ns) }\end{array}$ & $\begin{array}{c}\text { Carotenoid to Chl EET } \\
\text { efficiency \% }\end{array}$ & $\begin{array}{c}\text { Thermostability transition } \\
\text { temperature }\left({ }^{\circ} \mathrm{C}\right)\end{array}$ & $\begin{array}{l}\text { Photostability photobleaching rate } \\
\left(\Delta \% \text { Absorption }_{350-750 \mathrm{~mm}} \mathrm{~min}^{-1}\right)\end{array}$ \\
\hline$-\mathrm{LL}$ & $3.0 \pm 0.2$ & $90 \pm 1^{*}$ & $81 \pm 0.3$ & $-0.50 \pm 0.03^{*}$ \\
\hline
\end{tabular}

Fluorescence lifetime (438nm excitation, $680 \mathrm{~nm}$ detection, $n=4)$. Errors represent the $S D$. Carotenoid to $C h l$ excitation energy (EE) transfer (EET) efficiency, normalized to Chl a (assuming $100 \%$ transfer, $n=3$ ). Errors represent the SE (Figures $\mathbf{5 A}, \boldsymbol{B}$; Supplementary Figure $\mathbf{S 5}$ ). Thermostability (Figure $\mathbf{5 C}$ ). Photostability (Figure $\mathbf{5 D}, n=2)$. Errors represent the error of the fit. $n=$ number of biological replicates. $" p<0.05$.

photons $\mathrm{m}^{-2} \mathrm{~s}^{-1}$; Figure 5D). The results show that LHCII-LL has a higher temperature- and photostability (Table 2) than LHCII-HL.

\section{DISCUSSION}

Photoacclimation mechanisms are critical for the success of photosynthetic organisms and the xanthophyll cycles have proven to be major contributors to photoacclimation for many organisms. Thus far six xanthophyll cycles have been described but more may be present, especially among algae (GarcíaPlazaola et al., 2007). Here, we presented a new XC present in C. reinhardtii: the Loroxanthin cycle. We show its kinetics and its effects on the carotenoid composition of the antenna complexes (LhcbMs). The properties of the cycle are compared to those of the other XCs and are discussed in the context of its possible physiological role. 
A
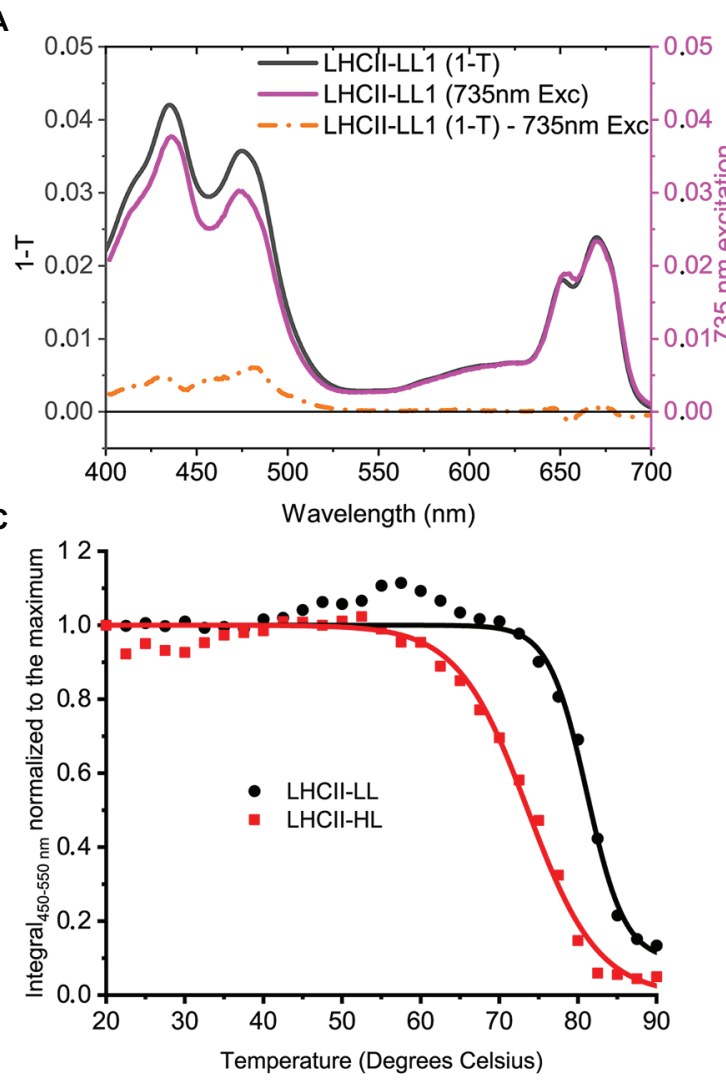

B

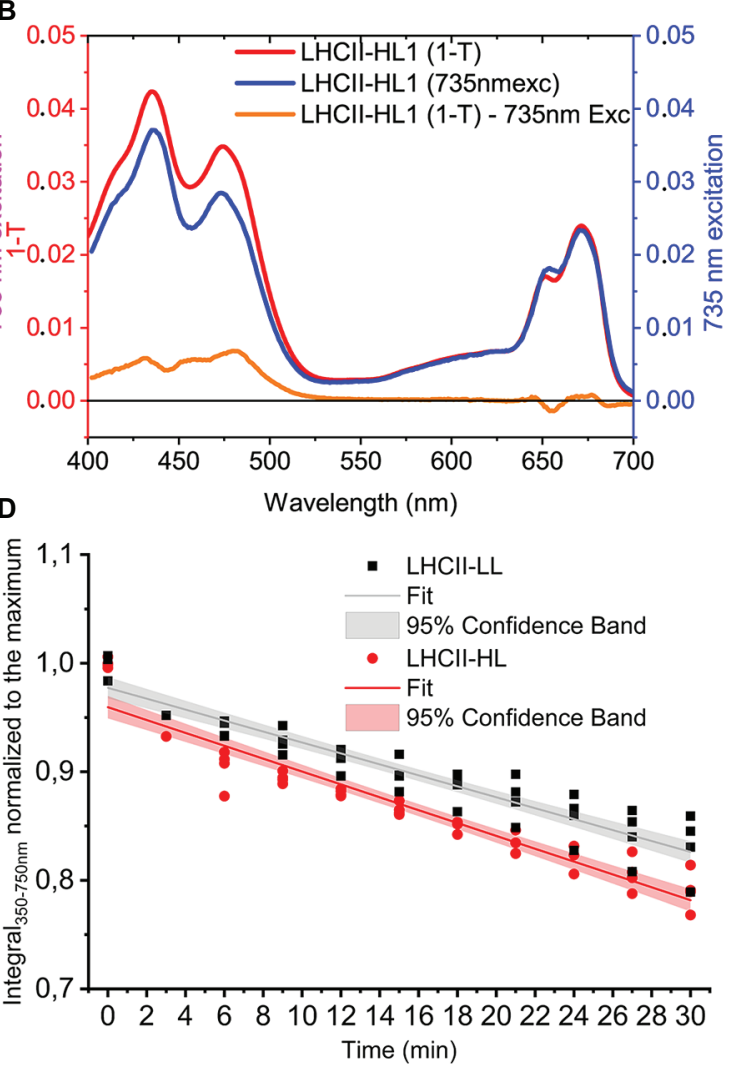

FIGURE 5 | Properties of LHCII-LL and LHCII-HL. (A; LHCII-LL) and (B; LHCII-HL) Carotenoid to Chl EET efficiency, tested by comparing the fitted 1-Transmission (1-T) and fluorescence excitation spectra normalized to the Chl a content (Full details in Supplementary Figures S3, S4) (C). Thermostability of LHCII-LL and LHCII-HL measured by the loss of the CD signal between 450 and $550 \mathrm{~nm}$ (the absolute area was used). The lines represent a sigmoidal fit of the data (D). Photostability of LHCII-LL and LHCII-HL tested by the photobleaching with a 4,500 $\mu \mathrm{mol}$ photons $\mathrm{m}^{-2} \mathrm{~s}^{-1}$ halogen lamp. Data from two biological and two technical replicates normalized to the average integral of the initial (maximum) absorption and fitted with a linear function.

\section{Changes in the Lutein Hydroxylation State Is a Long Term Acclimation Response in Chlamydomonas reinhardtii}

The Lo/(L+Lo) ratio changes slowly during a "simulated summer" day, following changes in light intensity. The amplitude of the change of the Lutein hydroxylation state is less than half of that observed after a sustained $(72 \mathrm{~h})$ change in light intensity (Figures 2E, 3C). The smaller value is mainly due to the larger amount of lutein in the culture upon $\mathrm{D} / \mathrm{N}$ cycle, during which the cells experience light stress and produce lutein, as observed before (Bonente et al., 2012; Polukhina et al., 2016; Nawrocki et al., 2020). Indeed, the maximum Lo/Chl ratio during $\mathrm{D} / \mathrm{N}$ cycle is $70 \%$ of that of the cells acclimated to LL for $72 \mathrm{~h}$. Because the change of the Lutein hydroxylation state is slow compared to the VAZ cycle in all the conditions tested, we conclude that the Loroxanthin cycle is a long-term acclimation process. This conclusion is in agreement with other data in C. reinhardtii (Pineau et al., 2001), showing that short term high light treatment had no significant effect on the Lo/ $(\mathrm{L}+\mathrm{Lo})$ ratio, and Scenedesmus Obliquus (Bishop et al., 1989), showing that the $\mathrm{Lo} /(\mathrm{L}+\mathrm{Lo})$ ratio changes by a factor of 4 upon a change in light intensity and was reversible in $48 \mathrm{~h}$.

\section{Loroxanthin Binding Sites in LHCII}

The pigment content of LhcbM trimers acclimated to HL or LL (Table 2) demonstrates that a change in the Lutein hydroxylation state is also reflected in the carotenoid composition of the LHCII trimers. The LhcbM of LL grown C. reinhardtii bind less Lutein than the Lhcb of plants and bind Loroxanthin instead, with small stoichiometrical differences among the LhcbMs (Natali and Croce, 2015). A change in the ratio between the LhcbMs would therefore not explain the change in the carotenoid composition of the trimers from low and high light acclimated cultures. Moreover, no large changes in LHCII composition were observed previously (Bonente et al., 2012).

The presence of 1.3 molecules of Loroxanthin per monomer in LHCII-LL indicates that this xanthophyll binds in at least two carotenoid binding sites. Out of the four carotenoid sites of the LhcbMs (Figure 1), Lo was suggested to bind to L1 because its presence influences the lowest energy Chls, known to be close to L1 (Natali and Croce, 2015). This effect is also visible in the complexes analyzed here (Figures 4B,D). The second binding site accommodating Loroxanthin is most probably L2, since in LHCII-HL Loroxanthin is almost entirely substituted by Lutein, and it 
is known that out of the remaining three binding sites (L2, $\mathrm{N} 1$, and V1) Lutein has the highest affinity for L2 (Croce et al., 1999). Moreover, several results seem to exclude that Loroxanthin is associated with $\mathrm{N} 1$ and V1 in a high amount: (i) the purification of LHC complexes via isoelectrofocusing largely removes the carotenoid bound in the V1 site, while it does not affect the carotenoids in L1 and L2 (Caffarri et al., 2001; Natali and Croce, 2015). Trimeric LHCII-LL isolated with this technique lose a large fraction of Violaxanthin and Lutein and much less Loroxanthin (Natali and Croce, 2015). Similar results were observed with native PAGE of solubilized thylakoids (Pineau et al., 2001). (ii) The N1 site of plant LHCII is highly specific for Neoxanthin and this is primarily due to the presence of a tyrosine forming an $\mathrm{H}$-bond with the $-\mathrm{OH}$ of the Neoxanthin (Caffarri et al., 2007). This tyrosine is conserved in all LhcbMs suggesting that the N1 site is specific to Neoxanthin also in these complexes. The fact that in LHCII-LL 0.8 Neoxanthin molecules are present supports this suggestion.

\section{Mechanistic Considerations of the Light-Dependent Change in Lutein and Loroxanthin Content}

Since Lutein in the L1 and L2 sites of LHCII is strongly bound to the complex, it is unlikely that an exchange of xanthophylls can occur in the folded complex. Also, the $\mathrm{OH}$ group of Loroxanthin is deeply buried in the transmembrane domain of LHCII and is thus unreachable from the outside. Thus, Loroxanthin and Lutein are most likely inserted in newly synthesized LHCII proteins during folding. In addition, the presence of only minimal quantities of Loroxanthin "free" in the membrane compared with Lutein and Violaxanthin (Pineau et al., 2001), suggests that the LhcbMs have a larger affinity for loroxanthin than for the other xanthophylls.

\section{Role of the Light-Dependent Change in Lutein and Loroxanthin Content}

The fact that the Lutein and Loroxanthin content in cells and LhcbM is related to light intensity changes suggests opposite roles for these two xanthophylls in light-harvesting and photoprotection. The excitation energy transfer efficiency of the carotenoids associated with LHCII is higher with Loroxanthin than Lutein $(5 \pm 1 \%)$. This increase is similar to that observed for Lutein-epoxide vs. Lutein (Matsubara et al., 2007) and increased excitation energy transfer efficiency of the carotenoids can lead to a growth advantage in low-light environments as observed for purple bacteria (Magdaong et al., 2014). On the other hand, Lutein seems to be important in high light conditions. One of the possibilities is that Lutein is a better $\mathrm{Chl}^{3}$ quencher or oxygen scavenger than Loroxanthin. However, this does not seem to be the case since the photostability of LHCII-LL is high and even slightly higher than that of LHCIIHL, meaning that both complexes are well protected. The thermostability of LHCII-LL is also increased compared to LHCII-HL and both complexes remain completely stable up to $60^{\circ} \mathrm{C}$ (Figure 5C), which is above the physiological temperature range of C. reinhardtii (Starks et al., 1981). The additional incorporation of Lutein in the LhcbM in HL might be important for NPQ.
Lutein has been shown to be involved in NPQ in vivo (Niyogi et al., 1997) and to quench chlorophyll singlet excited states directly in isolated complexes (Mascoli et al., 2019). The effectivity of quenching by loroxanthin might be lower and the availability of mutants of the Lutein hydroxylation enzyme would allow testing this hypothesis. The fact that the lifetime of LHCII in vitro does not change in the presence of Lutein or Loroxanthin is not conclusive in this respect because it is known that isolated LHCII are stable in their light-harvesting conformation (Ruban and Horton, 1992). However, the observation that NPQ can occur in the absence of Lutein and Loroxanthin (Niyogi et al., 1997) and even when non-native xanthophylls are associated with LHCII (Xu et al., 2020) suggests that the Lutein to Loroxanthin exchange might not have a significant effect on quenching in individual LHCs. Alternatively, the additional incorporation of Lutein in the LhcbM in HL could be a side-effect of the increase of Lutein content in the membrane upon high-light acclimation (Bonente et al., 2012; Polukhina et al., 2016) that may provide an increase in photoprotection that overcomes the disadvantages of additional Lutein binding to the LhcbMs.

\section{Comparison of the Loroxanthin Cycle With Other Xanthophyll Cycles}

The Loroxanthin cycle has some similarity with the LLx cycle of plants: (1) Lutein-epoxide has increased EET to Chl compared with Lutein $(+7.9 \%)$ and mostly binds to the Internal Lutein binding sites of LHCII (Matsubara et al., 2007). (2) Complete $\mathrm{LL}$ Lo/L and Lo/100 Chl $(a / b)$ ratios take more than $24 \mathrm{~h}$ to be attained, similar to the truncated LLx cycle (Esteban and García-Plazaola, 2014). However, there are also differences: (1) hydroxylation vs. epoxidation of Lutein; (2) The Loroxanthin cell content of LL grown C. reinhardtii is higher than the Lutein-epoxide content in more than $95 \%$ of the species that contain the LLx cycle (Esteban and García-Plazaola, 2014); and (3) the Loroxanthin content of LhcbM of C. reinhardtii $[(\mathrm{Lo} / \mathrm{L})=1]$ is higher than the Lutein-epoxide content of the Lhcb of shade plants $(\mathrm{Lx} / \mathrm{L}=.47-.8$; Matsubara et al., 2003, 2005; García-Plazaola et al., 2007).

The Loroxanthin cycle differs from the VAZ, in two main aspects: it is far slower and it leads to a change in the occupancy of the L1/L2 binding sites of LHCII, which is not the case for VAZ (Xu et al., 2015). Interestingly, the presence of both cycles in $C$. reinhardtii suggests that they are involved in slow and fast photoprotection strategies. A xanthophyll cycle operating at longer timescales than the VDE cycle, such as the LLx cycle (GarcíaPlazaola et al., 2007; Esteban and García-Plazaola, 2014) and the Loroxanthin cycle, is thus likely to provide evolutionary advantages for photosynthetic organisms that experience long periods of low light. Loroxanthin is present in algae of the Chlorophyte, Euglenophyte, and Chlorarachniophyte (Takaichi, 2011) and in addition to $C$. reinhardtii its content has been shown to fluctuate with the light intensity in the Chlorophytes Botryococcus braunii (van den Berg et al., 2019), Tetraselmis suecica (Garrido et al., 2009), and Scenedesmus obliquus (Senger et al., 1993). The loroxanthin cycle is therefore likely widespread and possibly active in all algae that contain Loroxanthin, similar to the LLx cycle in Lutein-epoxide containing plants (García-Plazaola et al., 2007). 


\section{DATA AVAILABILITY STATEMENT}

The raw data supporting the conclusions of this article will be made available by the authors, without undue reservation.

\section{AUTHOR CONTRIBUTIONS}

TB conceived the research and performed the experiments. TB and RC analyzed the data and wrote the manuscript. All authors contributed to the article and approved the submitted version.

\section{REFERENCES}

Allorent, G., Tokutsu, R., Roach, T., Peers, G., Cardol, P., Girard-Bascou, J., et al. (2013). A dual strategy to cope with high light in Chlamydomonas reinhardtii. Plant Cell 25, 545-557. doi: 10.1105/tpc.112.108274

Ballottari, M., Girardon, J., Dall'Osto, L., and Bassi, R. (2012). Evolution and functional properties of photosystem II light harvesting complexes in eukaryotes. Biochim. Biophys. Acta Bioenerg. 1817, 143-157. doi: 10.1016/j. bbabio.2011.06.005

Bassi, R., and Dall'Osto, L. (2021). Dissipation of light energy absorbed in excess: the molecular mechanisms. Annu. Rev. Plant Biol. 72, 47-76. doi: 10.1146/annurev-arplant-071720-015522

Bishop, N. I. (1996). The $\beta, \varepsilon$-carotenoid, lutein, is specifically required for the formation of the oligomeric forms of the light harvesting complex in the green alga, scenedesmus obliquus. J. Photochem. Photobiol. B Biol. 36, 279-283. doi: 10.1016/S1011-1344(96)07381-2

Bishop, N. I., Humbeck, K., Römer, S., and Senger, H. (1989). The mode of adaptation of the photosynthetic apparatus of a pigment mutant of Scenedesmus without light harvesting complex to different light intensities. J. Plant Physiol. 135, 144-149. doi: 10.1016/S0176-1617(89)80167-1

Bojko, M., Olchawa-Pajor, M., Goss, R., Schaller-Laudel, S., Strzałka, K., and Latowski, D. (2019). Diadinoxanthin de-epoxidation as important factor in the short-term stabilization of diatom photosynthetic membranes exposed to different temperatures. Plant Cell Environ. 42, 1270-1286. doi: 10.1111/ pce. 13469

Bonente, G., Ballottari, M., Truong, T. B., Morosinotto, T., Ahn, T. K., Fleming, G. R., et al. (2011). Analysis of LHcSR3, a protein essential for feedback de-excitation in the green alga Chlamydomonas reinhardtii. PLoS Biol. 9:e1000577. doi: 10.1371/journal.pbio.1000577

Bonente, G., Pippa, S., Castellano, S., Bassi, R., and Ballottari, M. (2012). Acclimation of Chlamydomonas reinhardtii to different growth irradiances. J. Biol. Chem. 287, 5833-5847. doi: 10.1074/jbc.M111.304279

Caffarri, S., Croce, R., Breton, J., and Bassi, R. (2001). The major antenna complex of photosystem II has a xanthophyll binding site not involved in light harvesting. J. Biol. Chem. 276, 35924-35933. doi: 10.1074/jbc.M105199200

Caffarri, S., Passarini, F., Bassi, R., and Croce, R. (2007). A specific binding site for neoxanthin in the monomeric antenna proteins CP26 and CP29 of Photosystem II. FEBS Lett. 581, 4704-4710. doi: 10.1016/j.febslet.2007.08.066

Caffarri, S., Tibiletti, T., Jennings, R., and Santabarbara, S. (2014). A comparison between plant photosystem I and photosystem II architecture and functioning. Curr. Protein Pept. Sci. 15, 296-331. doi: 10.217 4/1389203715666140327102218

Christa, G., Cruz, S., Jahns, P., de Vries, J., Cartaxana, P., Esteves, A. C., et al. (2017). Photoprotection in a monophyletic branch of chlorophyte algae is independent of energy-dependent quenching (qE). New Phytol. 214, 1132-1144. doi: $10.1111 / \mathrm{nph} .14435$

Chua, N., and Bennoun, P. (1975). Polypeptides of Chlamydomonas reinhardtii: wild-type and mutant strains deficient. Proc. Natl. Acad. Sci. U. S. A. 72, 2175-2179. doi: 10.1073/pnas.72.6.2175

Correa-Galvis, V., Redekop, P., Guan, K., Griess, A., Truong, T. B., Wakao, S., et al. (2016). Photosystem II subunit PsbS is involved in the induction of LHCSR protein-dependent energy dissipation in Chlamydomonas reinhardtii. J. Biol. Chem. 291, 17478-17487. doi: 10.1074/jbc.M116.737312

\section{ACKNOWLEDGMENTS}

Bart van Oort is acknowledged for helpful discussions.

\section{SUPPLEMENTARY MATERIAL}

The Supplementary Material for this article can be found online at: https://www.frontiersin.org/articles/10.3389/fpls.2022.797294/ full\#supplementary-material

Croce, R., Canino, G., Ros, F., and Bassi, R. (2002). Chromophore organization in the higher-plant photosystem II antenna protein CP26. Biochemistry 41, 7334-7343. doi: 10.1021/bi0257437

Croce, R., Cinque, G., Holzwarth, A. R., and Bassi, R. (2000). The soret absorption properties of carotenoids and chlorophylls in antenna complexes of higher plants. Photosynth. Res. 64, 221-231. doi: 10.1023/A:100645 5230379

Croce, R., and van Amerongen, H. (2020). Light harvesting in oxygenic photosynthesis: structural biology meets spectroscopy. Science 369:eaay2058. doi: 10.1126/science.aay2058

Croce, R., Weiss, S., and Bassi, R. (1999). Carotenoid-binding sites of the major light-harvesting complex II of higher plants. J. Biol. Chem. 274, 29613-29623. doi: 10.1074/jbc.274.42.29613

de Vries, J., and Archibald, J. M. (2018). Plant evolution: landmarks on the path to terrestrial life. New Phytol. 217, 1428-1434. doi: 10.1111/ nph. 14975

Dinc, E., Tian, L., Roy, L. M., Roth, R., Goodenough, U., and Croce, R. (2016). LHCSR1 induces a fast and reversible $\mathrm{pH}$-dependent fluorescence quenching in LHCII in Chlamydomonas reinhardtii cells. Proc. Natl. Acad. Sci. U. S. A. 113, 7673-7678. doi: 10.1073/pnas.1605380113

Drop, B., Webber-Birungi, M., Fusetti, F., Kourill, R., Redding, K. E., Boekema, E. J., et al. (2011). Photosystem I of Chlamydomonas reinhardtii contains nine light-harvesting complexes (Lhca) located on one side of the core. J. Biol. Chem. 286, 44878-44887. doi: 10.1074/jbc.M111.301101

Drop, B., Webber-Birungi, M., Yadav, S. K. N., Filipowicz-Szymanska, A., Fusetti, F., Boekema, E. J., et al. (2014). Light-harvesting complex II (LHCII) and its supramolecular organization in Chlamydomonas reinhardtii. Biochim. Biophys. Acta Bioenerg. 1837, 63-72. doi: 10.1016/j.bbabio.2013.07.012

Elrad, D., Niyogi, K. K., and Grossman, A. R. (2002). A major light-harvesting polypeptide of photosystem II functions in thermal dissipation. Plant Cell 14, 1801-1816. doi: 10.1105/tpc.002154

Esteban, R., and García-Plazaola, J. I. (2014). "Involvement of a second xanthophyll cycle in non-photochemical quenching of chlorophyll fluorescence: the lutein epoxide story," in Non-Photochemical Quenching and Energy Dissipation in Plants, Algae and Cyanobacteria, Advances in Photosynthesis and Respiration. Vol. 40. ed. B. Demmig-Adams (Dordrecht: Springer), 277-295.

Esteban, R., Matsubara, S., Jiménez, M. S., Morales, D., Brito, P., Lorenzo, R., et al. (2010). Operation and regulation of the lutein epoxide cycle in seedlings of Ocotea foetens. Funct. Plant Biol. 37, 859-869. doi: 10.1071/FP10014

Falkowski, P. G., and Chen, Y. (2003). "Photoacclimation of light harvesting systems in eukaryotic algae," in Light-Harvesting Antennas. eds. B. R. Green and W. W. Parson (Dordrecht: Springer), 423-447.

Fernández-Marín, B., Roach, T., Verhoeven, A., and García-Plazaola, J. I. (2021). Shedding light on the dark side of xanthophyll cycles. New Phytol. 230, 1336-1344. doi: 10.1111/nph.17191

Frank, H. A., Young, A. J., Britton, G., and Cogdell, R. J. (2004). The Photochemistry of Carotenoids. Springer Science \& Business Media.

García-Plazaola, J. I., Matsubara, S., and Osmond, B. (2007). The lutein epoxide cycle in higher plants: its relationships to other xanthophyll cycles and possible functions. Funct. Plant Biol. 34, 759-773. doi: 10.1071/fp07095

Garrido, J. L., Rodríguez, F., and Zapata, M. (2009). Occurrence of loroxanthin, loroxanthin decenoate, and loroxanthin dodecenoate in tetraselmis species (prasinophyceae, chlorophyta). J. Phycol. 45, 366-374. doi: 10.1111/j. 1529-8817.2009.00660.x 
Giordano, M., Beardall, J., and Raven, J. A. (2005). $\mathrm{CO}_{2}$ concentrating mechanisms in algae: mechanisms, environmental modulation, and evolution. Annu. Rev. Plant Biol. 56, 99-131. doi: 10.1146/annurev.arplant.56.032604.144052

Goss, R., Böhme, K., and Wilhelm, C. (1998). The xanthophyll cycle of Mantoniella squamata converts violaxanthin into antheraxanthin but not to zeaxanthin: consequences for the mechanism of enhanced non-photochemical energy dissipation. Planta 205, 613-621. doi: 10.1007/s004250050364

Goss, R., and Jakob, T. (2010). Regulation and function of xanthophyll cycledependent photoprotection in algae. Photosynth. Res. 106, 103-122. doi: $10.1007 / \mathrm{s} 11120-010-9536-\mathrm{x}$

Goss, R., and Latowski, D. (2020). Lipid dependence of xanthophyll cycling in higher plants and algae. Front. Plant Sci. 11:455. doi: 10.3389/fpls.2020.00455

Grossman, A. R., Lohr, M., and Im, C. S. (2004). Chlamydomonas reinhardtii in the landscape of pigments. Annu. Rev. Genet. 38, 119-173. doi: 10.1146/ annurev.genet.38.072902.092328

Gruszecki, W. I., and Strzałka, K. (2005). Carotenoids as modulators of lipid membrane physical properties. Biochim. Biophys. Acta Mol. basis Dis. 1740, 108-115. doi: 10.1016/j.bbadis.2004.11.015

Havaux, M. (1998). Carotenoids as membrane stabilizers in chloroplasts. Trends Plant Sci. 3, 147-151. doi: 10.1016/S1360-1385(98)01200-X

Havaux, M., Dall'Osto, L., and Bassi, R. (2007). Zeaxanthin has enhanced antioxidant capacity with respect to all other xanthophylls in Arabidopsis leaves and functions independent of binding to PSII antennae. Plant Physiol. 145, 1506-1520. doi: 10.1104/pp.107.108480

Havaux, M., and Niyogi, K. K. (1999). The violaxanthin cycle protects plants from photooxidative damage by more than one mechanism. Proc. Natl. Acad. Sci. U. S. A. 96, 8762-8767. doi: 10.1073/pnas.96.15.8762

Huang, Z., Shen, L., Wang, W., Mao, Z., Yi, X., Kuang, T., et al. (2021). Structure of photosystem I-LHCI-LHCII from the green alga Chlamydomonas reinhardtii in state 2. Nat. Commun. 12:1100. doi: 10.1038/s41467-021-21362-6

Jeffrey, S. W., Wright, S. W., and Zapata, M. (2011). "Microalgal classes and their signature pigments," in Phytoplankton Pigments: Characterization, Chemotaxonomy and Applications in Oceanography. eds. S. Roy, C. A. Llewellyn, E. S. Egeland and G. Johnsen (United Kingdom: Cambridge University Press), 3-77.

Johnson, M. P., Havaux, M., Triantaphylidès, C., Ksas, B., Pascal, A. A., Robert, B., et al. (2007). Elevated zeaxanthin bound to oligomeric LHCII enhances the resistance of Arabidopsis to photooxidative stress by a lipid-protective, antioxidant mechanism. J. Biol. Chem. 282, 22605-22618. doi: 10.1074/jbc. M702831200

Kirst, G. O., and Wiencke, C. (1995). Ecophysiology of polar algae. J. Phycol. 31, 181-199. doi: 10.1111/j.0022-3646.1995.00181.x

Kress, E., and Jahns, P. (2017). The dynamics of energy dissipation and xanthophyll conversion in Arabidopsis indicate an indirect photoprotective role of Zeaxanthin in slowly inducible and relaxing components of non-photochemical quenching of excitation energy. Front. Plant Sci. 8:2094. doi: 10.3389/fpls.2017.02094

Kromdijk, J., Głowacka, K., Leonelli, L., Gabilly, S. T., Iwai, M., Niyogi, K. K., et al. (2016). Improving photosynthesis and crop productivity by accelerating recovery from photoprotection. Science 354, 857-861. doi: 10.1126/science. aai 8878

Leliaert, F., Smith, D. R., Moreau, H., Herron, M. D., Verbruggen, H., Delwiche, C. F., et al. (2012). Phylogeny and molecular evolution of the green algae. Crit. Rev. Plant Sci. 31, 1-46. doi: 10.1080/07352689.2011.615705

Leonelli, L., Brooks, M. D., and Niyogi, K. K. (2017). Engineering the lutein epoxide cycle into Arabidopsis thaliana. Proc. Natl. Acad. Sci. U. S. A. 114, E7002-E7008. doi: 10.1073/pnas.1704373114

Lepetit, B., Volke, D., Gilbert, M., Wilhelm, C., and Goss, R. (2010). Evidence for the existence of one antenna-associated, lipid-dissolved and two proteinbound pools of diadinoxanthin cycle pigments in diatoms. Plant Physiol. 154, 1905-1920. doi: 10.1104/pp.110.166454

Liu, Z., Yan, H., Wang, K., Kuang, T., Zhang, J., Gui, L., et al. (2004). Crystal structure of spinach major light-harvesting complex at 2.72 A resolution. Nature 428, 287-292. doi: 10.1038/nature02373

Magdaong, N. M., Lafountain, A. M., Greco, J. A., Gardiner, A. T., Carey, A. M., Cogdell, R. J., et al. (2014). High efficiency light harvesting by carotenoids in the LH2 complex from photosynthetic bacteria: unique adaptation to growth under low-light conditions. J. Phys. Chem. B 118, 11172-11189. doi: 10.1021/jp5070984

Mascoli, V., Liguori, N., Xu, P., Roy, L. M., van Stokkum, I. H. M., and Croce, R. (2019). Capturing the quenching mechanism of light-harvesting complexes of plants by zooming in on the ensemble. Chem 5, 2900-2912. doi: 10.1016/j.chempr.2019.08.002

Matsubara, S., Chen, Y. C., Caliandro, R., Govindjee, G., and Clegg, R. M. (2011). Photosystem II fluorescence lifetime imaging in avocado leaves: contributions of the lutein-epoxide and violaxanthin cycles to fluorescence quenching. J. Photochem. Photobiol. B Biol. 104, 271-284. doi: 10.1016/j. jphotobiol.2011.01.003

Matsubara, S., Morosinotto, T., Bassi, R., Christian, A. L., Fischer-Schliebs, E., Lüttge, U., et al. (2003). Occurrence of the lutein-epoxide cycle in mistletoes of the Loranthaceae and Viscaceae. Planta 217, 868-879. doi: 10.1007/ s00425-003-1059-7

Matsubara, S., Morosinotto, T., Osmond, C. B., and Bassi, R. (2007). Short- and long-term operation of the lutein-epoxide cycle in light-harvesting antenna complexes. Plant Physiol. 144, 926-941. doi: 10.1104/pp.107.099077

Matsubara, S., Naumann, M., Martin, R., Nichol, C., Rascher, U., Morosinotto, T., et al. (2005). Slowly reversible de-epoxidation of lutein-epoxide in deep shade leaves of a tropical tree legume may "lock-in" lutein-based photoprotection during acclimation to strong light. J. Exp. Bot. 56, 461-468. doi: 10.1093/ jxb/eri012

Minagawa, J., and Takahashi, Y. (2004). Structure, function and assembly of photosystem II and its light-harvesting proteins. Photosynth. Res. 81, 241-263. doi: 10.1007/s11120-004-2079-2

Mozzo, M., Dall'Osto, L., Hienerwadel, R., Bassi, R., and Croce, R. (2008). Photoprotection in the antenna complexes of photosystem II: role of individual xanthophylls in chlorophyll triplet quenching. J. Biol. Chem. 283, 6184-6192. doi: $10.1074 /$ jbc.M708961200

Mozzo, M., Mantelli, M., Passarini, F., Caffarri, S., Croce, R., and Bassi, R. (2010). Functional analysis of photosystem I light-harvesting complexes (Lhca) gene products of Chlamydomonas reinhardtii. Biochim. Biophys. Acta Bioenerg. 1797, 212-221. doi: 10.1016/j.bbabio.2009.10.005

Natali, A., and Croce, R. (2015). Characterization of the major light-harvesting complexes (LHCBM) of the green alga Chlamydomonas reinhardtii. PLoS One 10:e119211. doi: 10.1371/journal.pone.0119211

Nawrocki, W. J., Liu, X., and Croce, R. (2020). Chlamydomonas reinhardtii exhibits de facto constitutive NPQ capacity in physiologically relevant conditions. Plant Physiol. 182, 472-479. doi: 10.1104/pp.19.00658

Nawrocki, W. J., Santabarbara, S., Mosebach, L., Wollman, F. A., and Rappaport, F. (2016). State transitions redistribute rather than dissipate energy between the two photosystems in Chlamydomonas. Nat. Plants 2, 16031-16037. doi: 10.1038/NPLANTS.2016.31

Niyogi, K. K., Björkman, O., and Grossman, R. (1997). The roles of specific xanthophylls in photoprotection. Proc. Natl. Acad. Sci. U. S. A. 94, 14162-14167. doi: $10.1073 /$ pnas.94.25.14162

Pan, X., Cao, P., Su, X., Liu, Z., and Li, M. (2020). Structural analysis and comparison of light-harvesting complexes I and II. Biochim. Biophys. Acta Bioenerg. 1861:148038. doi: 10.1016/j.bbabio.2019.06.010

Paulsen, H., Finkenzeller, B., and Kühlein, N. (1993). Pigments induce folding of light-harvesting chlorophyll $a / b$-binding protein. Eur. J. Biochem. 215, 809-816. doi: 10.1111/j.1432-1033.1993.tb18096.x

Peers, G., Truong, T. B., Ostendorf, E., Busch, A., Elrad, D., Grossman, A. R., et al. (2009). An ancient light-harvesting protein is critical for the regulation of algal photosynthesis. Nature 462, 518-521. doi: 10.1038/ nature 08587

Perera, I., Subashchandrabose, S. R., Venkateswarlu, K., Naidu, R., and Megharaj, M. (2018). Consortia of cyanobacteria/microalgae and bacteria in desert soils: an underexplored microbiota. Appl. Microbiol. Biotechnol. 102, 7351-7363. doi: 10.1007/s00253-018-9192-1

Pineau, B., Gérard-Hirne, C., and Selve, C. (2001). Carotenoid binding to photosystems I and II of Chlamydomonas reinhardtii cells grown under weak light or exposed to intense light. Plant Physiol. Biochem. 39, 73-85. doi: 10.1016/S0981-9428(00)01215-8

Polukhina, I., Fristedt, R., Dinc, E., Cardol, P., and Croce, R. (2016). Carbon supply and photoacclimation cross talk in the green alga Chlamydomonas reinhardtii. Plant Physiol. 172, 1494-1505. doi: 10.1104/pp.16.01310

Quaas, T., Berteotti, S., Ballottari, M., Flieger, K., Bassi, R., Wilhelm, C., et al. (2015). Non-photochemical quenching and xanthophyll cycle activities in six green algal species suggest mechanistic differences in the process of excess energy dissipation. J. Plant Physiol. 172, 92-103. doi: 10.1016/j. jplph.2014.07.023 
Raniello, R., Lorenti, M., Brunet, C., and Buia, M. C. (2006). Photoacclimation of the invasive alga Caulerpa racemosa var. cylindracea to depth and daylight patterns and a putative new role for siphonaxanthin. Mar. Ecol. 27, 20-30. doi: 10.1111/j.1439-0485.2006.00080.x

Reinhold, C., Niczyporuk, S., Beran, K. C., and Jahns, P. (2008). Short-term down-regulation of zeaxanthin epoxidation in Arabidopsis thaliana in response to photo-oxidative stress conditions. Biochim. Biophys. Acta Bioenerg. 1777, 462-469. doi: 10.1016/j.bbabio.2008.03.002

Rmiki, N.-E., Brunet, C., Cabioch, J., and Lemoine, Y. (1996). "XanthophyllCycle and Photosynthetic Adaptation to Environment in Macro- and Micro Algae," in Developments in Hydrobiology - Fifteenth International Seaweed Symposium. January 1995, Valdivia, Chile, 407-413.

Ruban, A. V., and Horton, P. (1992). Mechanism of $\Delta \mathrm{pH}$-dependent dissipation of absorbed excitation energy by photosynthetic membranes. I. Spectroscopic analysis of isolated light-harvesting complexes. BBA-Bioenergetics 1102, 30-38. doi: 10.1016/0005-2728(92)90061-6

Ruban, A. V., Pascal, A. A., and Robert, B. (2000). Xanthophylls of the major photosynthetic light-harvesting complex of plants: identification, conformation and dynamics. FEBS Lett. 477, 181-185. doi: 10.1016/S0014-5793(00)01799-3

Sasso, S., Stibor, H., Mittag, M., and Grossman, A. R. (2018). From molecular manipulation of domesticated Chlamydomonas reinhardtii to survival in nature. Elife 7, 1-14. doi: 10.7554/elife.39233

Stange, C. (ed.) (2016). Carotenoids in Nature: Biosynthesis, Regulation and Function. Springer.

Senger, H., Wagner, C., Hermsmeier, D., Hohl, N., Urbig, T., and Bishop, N. I. (1993). The influence of light intensity and wavelength on the contents of a- and b-carotene and their xanthophylls in green algae. J. Photochem. Photobiol. B Biol. 18, 273-279. doi: 10.1016/1011-1344(93)80075-K

Shen, L., Huang, Z., Chang, S., Wang, W., Wang, J., Kuang, T., et al. (2019). Structure of a C2S2M2N2-type PSII-LHCII supercomplex from the green alga Chlamydomonas reinhardtii. Proc. Natl. Acad. Sci. U. S. A. 116, 21246-21255. doi: $10.1073 /$ pnas.1912462116

Snyder, A. M., Clark, B. M., and Bungard, R. A. (2005). Light-dependent conversion of carotenoids in the parasitic angiosperm Cuscuta reflexa $\mathrm{L}$. Plant Cell Environ. 28, 1326-1333. doi: 10.1111/j.1365-3040.2005.01379.x

Stamenković, M., Bischof, K., and Hanelt, D. (2014). Xanthophyll cycle pool size and composition in several cosmarium strains (Zygnematophyceae, Streptophyta) are related to their geographic distribution patterns. Protist 165, 14-30. doi: 10.1016/j.protis.2013.10.002

Starks, T. L., Shubert, L. E., and Trainor, F. R. (1981). Ecology of soil algae: a review. Phycologia 20, 65-80. doi: 10.2216/i0031-8884-20-1-65.1

Sueoka, N. (1960). Mitotic replication of deoxyribonucleic acid in Chlamydomonas reinhardi. Proc. Natl. Acad. Sci. U. S. A. 46, 83-91. doi: 10.1073/pnas.46.1.83

Suga, M., Ozawa, S.-I., Yoshida-Motomura, K., Akita, F., Miyazaki, N., and Takahashi, Y. (2019). Structure of the green algal photosystem I supercomplex with a decameric light-harvesting complex I. Nat. Plants 5, 626-636. doi: 10.1038/s41477-019-0438-4

Takaichi, S. (2011). Carotenoids in algae: distributions, biosyntheses and functions. Mar. Drugs 9, 1101-1118. doi: 10.3390/md9061101

Tibiletti, T., Auroy, P., Peltier, G., and Caffarri, S. (2016). Chlamydomonas reinhardtii PsbS protein is functional and accumulates rapidly and transiently under high light. Plant Physiol. 171, 2717-2730. doi: 10.1104/ pp.16.00572

van den Berg, T. E., Chukhutsina, V. U., van Amerongen, H., Croce, R., and van Oort, B. (2019). Light acclimation of the colonial green alga Botryococcus braunii strain showa. Plant Physiol. 179, 1132-1143. doi: 10.1104/pp.18.01499

van den Berg, T. E., van Oort, B., and Croce, R. (2018). Light-harvesting complexes of Botryococcus braunii. Photosynth. Res. 135, 191-201. doi: 10.1007/ s11120-017-0405-8

Van Oort, B., Amunts, A., Borst, J. W., Van Hoek, A., Nelson, N., Van Amerongen, H., et al. (2008). Picosecond fluorescence of intact and dissolved PSI-LHCI crystals. Biophys. J. 95, 5851-5861. doi: 10.1529/biophysj.108.140467

Wobbe, L., Bassi, R., and Kruse, O. (2016). Multi-level light capture control in plants and green algae. Trends Plant Sci. 21, 55-68. doi: 10.1016/j. tplants.2015.10.004

Xu, P., Chukhutsina, V. U., Nawrocki, W. J., Schansker, G., Bielczynski, L. W., Lu, Y., et al. (2020). Photosynthesis without $\beta$-carotene. Elife 9:e58984. doi: 10.7554/ELIFE.58984

Xu, P., Tian, L., Kloz, M., and Croce, R. (2015). Molecular insights into Zeaxanthin-dependent quenching in higher plants. Sci. Rep. 5:13679. doi: $10.1038 /$ srep 13679

Conflict of Interest: The authors declare that the research was conducted in the absence of any commercial or financial relationships that could be construed as a potential conflict of interest.

Publisher's Note: All claims expressed in this article are solely those of the authors and do not necessarily represent those of their affiliated organizations, or those of the publisher, the editors and the reviewers. Any product that may be evaluated in this article, or claim that may be made by its manufacturer, is not guaranteed or endorsed by the publisher.

Copyright (c) 2022 van den Berg and Croce. This is an open-access article distributed under the terms of the Creative Commons Attribution License (CC BY). The use, distribution or reproduction in other forums is permitted, provided the original author(s) and the copyright owner(s) are credited and that the original publication in this journal is cited, in accordance with accepted academic practice. No use, distribution or reproduction is permitted which does not comply with these terms. 